\title{
LANGUAGE USE IN ASYNCHRONOUS COMPUTER-MEDIATED COMMUNICATION IN TAIWAN
}

\author{
Daphne Li-jung Huang, Providence University, Taiwan \\ Daphne Huang is currently teaching at Providence University in Taiwan. She \\ completed her doctoral thesis at University of Melbourne, Australia, in the fields \\ of bilingualism and computer-mediated communication. Her main research interest \\ continues from her doctoral thesis in bilingualism in computer-mediated commu- \\ nication. She is also interested in sociolinguistics and cross-cultural communication. \\ Correspondence to Daphne Li-jung Huang: ljhuang@pu.edu.tw
}

\begin{abstract}
This paper describes how Chinese-English bilinguals in Taiwan use their languages in asynchronous computer-mediated communication, specifically, via Bulletin Board System (BBS) and email. The main data includes two types: emails collected from a social network and postings collected from two BBS websites. By examining patterns of language choice and language use in these two types of data, the aim of this research is to investigate how a language contact phenomenon - code-switching (CS) - is realized via different writing systems in online environment.

The data reveals that there are two levels of CS: CS between languages and CS between writing systems. In terms of CS between languages, three patterns are found: CS between Mandarin and Taiwanese, CS between Mandarin and English, CS between Mandarin, Taiwanese and English. In terms of CS between writing systems, three patterns are also identified: CS between Standard Written Chinese (SWC) and Zhuyin, CS between SWC and English alphabet, and CS between SWC, Zhuyin and English alphabet. This paper presents the two levels of CS by using the examples found in the data and describes the complex relationship between different writing systems and different languages. It is concluded that CS is found to be a common phenomenon on asynchronous $\mathrm{CMC}$, where it fosters multilingualism and multi-orthography.
\end{abstract}

\section{INTRODUCTION}

Language contact is a widespread phenomenon in most parts of the world and it has thus become one of the most researched areas of linguistics in general and bilingualism in specific in the past two decades. Language contact phenomena include code-switching, code-mixing, borrowing, and transference. Because of the interest in language contact and specifically in code-switching (abbreviated as CS hereafter), the literature has witnessed descriptions of different bilingual ${ }^{1}$ communities and studies of contact between 
different language pairs. Within the studies of CS, spoken interaction has been the main focus, while written interaction such as letter writing, dairy writing and email writing has been largely ignored in the literature. As the use of Internet, which facilitates written interaction, has become more and more common, computer-mediated communication (CMC) has received much attention from linguists who are now more interested in studying language use via CMC in bilingual communities (e.g. Paolillo, 1999, 2001; Su, 2003; Hinrichs, 2006). Written interaction has thus become an important area which requires further study. To complement the field of bilingualism in CMC context, it is the aim of this paper to describe CS in two forms of CMC mediums - bulletin board system (BBS) and email. This paper is based on two previous research projects: one examining language choice and language use in emails by a group of Chinese-English bilinguals (Huang, 2004); and the other on language use and CS in BBS (Huang, 2008). The main data includes two types: emails collected from a Chinese-English bilingual social network in Taiwan and postings collected from two Taiwanese BBS websites. By examining patterns of language choice and language use in these two types of data, this paper describes how CS is realized via different writing systems in online environment. The languages considered in this study include Mandarin, Taiwanese and English and the writing systems considered include Standard Written Chinese (SWC), Chinese Zhuyin (also known as 'Mandarin phonetic symbols'), and English alphabet.

Accordingly, the main aim of this research is to address two major issues: 1) the adaptations of writing systems in online environment and 2) language choice in multilingual contexts in order to further our understanding of how bilinguals use their linguistic repertoires in online environment (see Danet \& Herring, 2003). This can give us some insights about the influence of the new mediums of CMC on the language use of bilinguals.

\section{BACKGROUND LITERATURE}

This section provides a brief description of studies in the areas of CS and CMC. The focus is on defining basic terminology and describing important concepts for the interpretation of the data.

\subsection{CODE-SWITCHING (CS)}

CS has been studied extensively (e.g. Auer, 1984, Auer, 1998; Clyne, 1991; Gumperz, 1982; Heller, 1988; Milroy \& Muysken, 1995; Muysken, 2000, among others) since Haugen (1953) first introduced the term switching to refer to any instances in which the 
speaker stops using language A and uses language B. Following Haugen, Gumperz (1982, p. 59) further defines CS as 'the juxtaposition within the same speech exchange of passages of speech belonging to two different grammatical systems of sub-systems'. In Gumperz's original definition, CS refers to different linguistic systems, that is, to different languages. This definition was later extended to cover the alternate use of different varieties of languages (Myers-Scotton, 1993; Myers-Scotton, 1998).

The term CS is often contrasted with 'borrowing' depending on the integration of an item from one language to the other (see e.g. Myers-Scotton, 1993). If an item is said to be integrated to the system of another language, it is considered as 'borrowing'. Considering the lexical item 'resume', it is considered as an English word if the pronunciation is based on the English phonological system. However, it would be considered as an instance of CS if the word is pronounced in French. In addition, the term CS has been contrasted with 'code-mixing' based on two criteria: degree of integration and unconscious choice. Code-mixing often implies a certain degree of integration and it is sometimes considered as a code itself. That is, the languages involved cannot be distinguished and such mixing is considered as one code. As can be seen, together with the popularity of the topic, confusion has been created on the use of the terminology (e.g. see Clyne, 1987). For the purpose of this study, CS is used to cover any instances of 'the alternate use of two or more languages or writing systems in a discourse'. No distinction is made between CS, code-mixing, and borrowing. The written data (both BBS and email) shows that, firstly, integration is not applicable since we are dealing with completely different writing systems. Secondly, in contrast to conversational CS which is often considered to be rapid, largely unconscious, and used for communicative effect, CS in BBS and email writing is a conscious choice since it entails a physical switch when changing the typing systems (see Section 3). For these two reasons, the term CS is used as a cover term and no differentiation is made between CS, code-mixing and borrowing.

\subsection{COMPUTER-MEDIATED COMMUNICATION (CMC)}

Communication via Internet is referred to as computer-mediated communication. It is defined as communication that takes place between human beings via the instrumentality of computers (Herring, 1996), which can be further divided into synchronous and asynchronous CMC in terms of time delay. In terms of forms, CMC can be divided into text-based, audio-visual, and mixed forms (see e.g. Hine, 2000; Murray, 1995). With the popularisation of the Internet, its impact on our everyday lives has been enormous and CMC has become one of the most researched areas nowadays (e.g. Crystal, 2001; Danet \& Herring, 2007; Hinrichs, 2006; Warschauer 2006; as well as research articles 
in journals such as Language Learning and Technology and Journal of Computer-Mediated Communication). Discourse in CMC has been studied and recognized as exhibiting unique styles of communication, rendering the term computer-mediated discourse, encompassing all kinds of interpersonal communication carried out on the Internet, e.g., by email, instant messaging, web discussion boards, and chat channels (Herring, 2001; Herring, 2004). It should be noted that the focus is on 'human communication', not the medium. In other words, CMC users employ the medium to achieve their communication purposes by exploiting their linguistic resources. Specifically, writing systems in CMC are adapted to compensate for their inherent inadequacies such as lack of facial expressions, posture, dress, social status indicators, and vocal cues (Sproull \& Kiesler, 1991).

Previous studies in CMC have focused on monolingual communities where various styles of the same language are used (e.g. Baron, 2000; Crystal, 2001). There has been growing attention on how bilinguals use their linguistic resources in online environment. For example, Journal of Computer-mediated Communication has dedicated an issue on the topic of 'The multilingual internet' with a focus on language use in instant messaging, email and chat. This issue includes discussions in four areas: 1) adaptations of writing systems in online environments; 2) social interpretations of, and attitudes toward, such adaptation; 3) language choice in multilingual contexts; and 4) gender and language dynamics in CMC in non-English-speaking cultures (Danet \& Herring, 2003). This issue has provided important resources in providing a link between bilingualism and CMC. With similar goals in mind, this paper addresses issues such as the adaptations of writing systems in online environment and language choice in multilingual contexts. The following section will briefly introduce the two selected CMC mediums as well as studies in these areas.

\subsubsection{EMAIL AND BULLETIN BOARD SYSTEM (BBS)}

Among the various mediums offered by the Internet, email has become one of the main mediums since the 1990s (see Baron, 2000). It is a form of asynchronous Internet communication, which allows text messages to be sent to specific address(es) (Hine, 2000). Email communication allows people to type extended messages at computer terminals and to have those messages electronically transmitted to recipients who can read, reply, delete, print, forward, or file them (Murray, 1995) at marginal cost. Emails also reduce the distance between speakers from different or same linguistic backgrounds, as well as providing easier access for intercultural communication (Baron, 2000). The Ministry of Transportation and Communication (MOTC) of Taiwan conducted a survey in 2001 and it was found that $80 \%$ of people logging on the Internet have email account(s). 
Among these people, $51 \%$ of people had more than one email account. This indicates the popularity of email use in Taiwan. In terms of length of time using the Internet, 39\% of interviewees in this survey had been using the Internet for more than three years, and $21 \%$ between 1 to 2 years, with an average length of 2.2 years. The report also found that email use is one of the most popular functions of Internet. According to a recent market survey conducted by Taiwan Network Information Center (TWNIC), there is more than $60 \%$ of the Taiwanese population using the Internet regularly by June, 2006, of which the email and BBS has become one of the most popular functions (Ishii \& Wu, 2006).

The term BBS currently may be used to refer to any online forum or message board. A BBS is mainly used for meeting people and having discussions in message boards as well as for publishing articles, downloading software, playing games and many more things (Wikipedia, 2007). It is a type of asynchronous CMC where users do not have to be in front of their computer terminals at the same time. Users can post messages on a public forum or send messages to a particular user. BBSs offer integrated services and the main function of a BBS is to share or exchange messages or other files on a network. Typical BBS features include:

- bulletins: information postings;

- local mail: messages which can be directed to individual users on the same BBS;

- local message forums: topical areas where messages on a given topic are posted publicly;

- net mail: forums that are actively forwarded to other member BBS so that the messages are available on any BBS subscribing to the network;

- echo mail: similar to net mail, but using a different system known as QWK; and

- file downloads: files available on the BBSs which users can transfer to their computers (Henry, 2002).

\subsubsection{STUDIES IN BILINGUALISM AND COMPUTER-MEDIATED COMMUNICATION}

Due to the popularity of the computer medium, there has been a large and growing literature on CMC. However, previous research often focuses on describing its impact on the use of spoken or written forms of language (e.g. Crystal, 2001; Danet, 2001). More recently, there has been a growing interest in how bilinguals use their languages on the Internet. For instance, Warschauer, El Said \& Zohry (2002) investigated language use in a bilingual community where a group of young professionals between the ages of 24 to 36 speaking both English and Arabic interact in online communication in Egypt. Their 
study indicates that, among this group of young professionals, English is used overwhelmingly not only on the Internet, but also in formal email communication $(92.5 \%)$. Moreover, they found that classical Arabic in Arabic script, the usual script for Arabic, was seldom used in their participants' Internet communication. Instead, a romanized version of Egyptian Arabic was used in informal email messages and online chats. Lam (2004) examined the social and discursive practices in a Chinese/English bilingual chatroom and how the Internet chatroom provides a context of language socialization for two teenage Chinese immigrants in the US. Lam found that a mixed-code variety of English is adopted and developed among the two teenagers. This mixed-code variety includes different degrees of CS, ranging from predominantly English to predominantly Cantonese. Such mixing is interpreted as developing immigrant dual identities. Hinrichs (2006) investigated the use of Creole and English among a group of university Jamaican students living in the UK. He found that the use of vernacular codes in written CS practices is an important marker of informality. Further, the stigmatized, experimental, and novel aspects of the non-standard code (Creole) in writing are employed in contrast with the standard code (English), and serve as additional expressive resources which give writers the change of greater expressive wealth than in monolingual settings. Studies on BBSs have been scarce, especially in bilingual communities. Su (2003) investigates the use of the writing systems on two BBSs of two college student organizations in Taiwan, in which she identifies four creative uses of writing systems: the rendering in Chinese characters of the sounds of English, Taiwanese, and Taiwanese-accented Mandarin, and the recycling of a transliteration alphabet (i.e. Zhuyin) used in Taiwan. She argues that the written nature of the Internet enables these practices, and that the everyday meanings associated with the writing systems and languages are appropriated and reproduced through online practice, resulting in a unique mode of communication in its own right.

Writings in email and BBS resemble oral speech in certain aspects, and users communicate, provide and get information as if they are in a face-to-face conversation situation (Crystal, 2001). In connection to the above-mentioned studies, this paper considers a language contact phenomenon in relation to the interactivity of the medium. That is, the fact that CMC offers bilinguals a new medium where informality and interactivity prevail may exert an influence on their language choice and language use. This study aims to link the field of CS and CMC in order to fill the gap in the research. 


\section{LINGUISTIC SKETCH OF TAIWAN}

Taiwan is a multilingual and multiethnic community. There are more than 20 native languages in Taiwan, including Hakka, Taiwanese, and many indigenous languages (Grimes, 1996). The Taiwanese population is mainly composed of four ethnic groups: Austronesian aborigines (1.7\%), Hakka (12\%), Mainlanders (13\%), and Holo (referring to 'Taiwanese'3 in this paper) $(73.3 \%)$ (Huang, 1993).

\subsection{LINGUISTIC SKETCH OF SPOKEN LANGUAGES IN TAIWAN: MANDARIN, TAIWANESE AND ENGLISH}

There are two major spoken languages used in Taiwan: Mandarin and Taiwanese. They differ in phonology, lexicon, and in the tones. Mandarin is the official language in Taiwan, established by the Kuomintang government (Hsiau, 1997). Since then, Mandarin has been the language of education, government, business interaction (to some extent) and it is often used in more formal settings. Taiwanese is the native language of about $70 \%$ of the population of Taiwan. In addition to Mandarin and Taiwanese, there has been an increasing use of English in various writings, including novels, magazines, and newspapers. The popularity of English in Taiwan is in conjunction with the importance of English as the global language (Crystal, 1997). In order to promote the internationalization of Taiwan, the Ministry of Education has extended the teaching of English to include the primary school level. All fifth and sixth grade students in Taiwan are thus required to study English as part of their formal education (Government Information Office, 2002). This is further extended to third grade students since 2005 (Chao, 2007). Today, the use of English is recognised as an economical advantage and such pragmatic view has led to the increasing use of English in writings.

\subsection{WRITING SYSTEMS IN TAIWAN: STANDARD WRITTEN CHINESE, CHINESE ZHUYIN, AND ENGLISH ALPHABET}

This section turns the discussion to the use of writing systems in Taiwan, focusing on the relationship between the writing systems and the languages represented by these writing systems. Chinese is a logographic language and the written form used today is often referred to as Standard Written Chinese (SWC). The SWC considered here refers to the 'full' script used in Taiwan (as opposed to the 'simplified' script used in People Republic of China). There are three writing systems currently being used in Taiwan: SWC, Chinese Zhuyin and English alphabet. SWC is mainly used to represent Mandarin while Zhuyin is used to aid learning the Mandarin pronunciation. The Zhuyin system 
uses 37 special symbols to represent the Mandarin sounds. These symbols are printed next to the Chinese characters in children's books to help them match the pronunciations with the characters. Nowadays, Zhuyin can be used to represent the pronunciations of both Mandarin and Taiwanese. Since there is not yet a standardized writing system for Taiwanese (Chiung, 2001; Tiu, 1998), the Taiwanese pronunciation can be represented by either SWC or Zhuyin. In addition, the use of the English alphabet has become more and more common in various domains such as magazines, newspapers, novels and so on, since the promotion of English as the first foreign language in Taiwan. Generally speaking, both SWC and Zhuyin are used to represent Mandarin while English alphabet is used to represent English. However, it is possible to use English alphabet to represent Mandarin and Taiwanese pronunciations and SWC and Zhuyin to present English pronunciation. Taiwanese is often represented by SWC and Zhuyin; however, such use is not conventional as it is not standardized in writing yet. Furthermore, Zhuyin can be used as one of the Chinese keyboard input methods in computers. The more common input methods used in Taiwan include Zhuyin, Cangiie, Dayi, and Boshiami. Zhuyin input method makes use of the pronunciation. It should be noted that a physical act is required when the writer wishes to change the typing systems, regardless of the Chinese input method being used. The writer can have it configured to any key combination on the computer keyboard or alternatively to use the mouse to switch the typing systems. Based on the various writing systems available to the CMC users, this paper will show that CS is a common practice to achieve linguistic communication.

\section{METHODOLOGY}

The purpose of this research is to describe how Chinese-English bilinguals in Taiwan use their languages in asynchronous CMC, focusing on BBS and email. The main data is drawn from the two mediums, including emails collected from a social network and postings collected from two BBSs. The first set of data includes 223 emails written among a network of eight Chinese-English bilinguals in Taiwan, who volunteered to participate in a previous project. The second set of data includes postings collected from two BBSs - http://www.ptt.cc and http://bbs.nsysu.edu.tw. The former (PTT BBS) is used predominantly by students from National Taiwan University and the latter (NTC BBS) by students from National Sun Yat-Sen University. These two websites were selected because they are considered to be very popular BBS websites among university students. According to the information from 'Immediate Hot Boards' automatically rating the most frequently used BBS in Taiwan, both PPT BBS and NTC BBS are rated as the 'hottest' boards. By 
examining patterns of language choice and language use in these two types of data, the aim of this research is to investigate how CS is realized via different writing systems in online environment.

\subsection{PARTICIPANTS AND DATA COLLECTION}

For the email data, the participants were recruited through snowballing. 'Friends of friends' (Milroy, 1987) were told about the project and then volunteered to participate in the study. The participants include eight native Chinese speakers, speaking Mandarin and/or Taiwanese as their first language(s), and aged between 25-40 years. The corpus of 223 emails was collected via a forwarded email system in the period of 2001 to 2003. Emails written to and from one selected participant (by volunteer) were collected by her and then forwarded to the researcher. These emails were then grouped together according to the content.

For the BBS data, postings were accessed and collected from 2006 to 2007, including postings, and comments or response postings, which discuss the posters' daily activities, gossips, sports, schools, feelings, jobs, and fashion, from a variety of boards. These messages are stored on BBS sites which can be visited, read, and retrieved by users, available to the general public. Five postings were collected per week randomly from each BBS site, making up to a corpus of 1040 postings in total.

Based on the two sets of data, tokens of switches were first identified and then categorised in terms of its structural level. Two levels of CS were distinguished: single word CS and inter-clausal CS. Single word switches were then first classified into different lexical categories and inter-clausal switches were classified into CS at the clause periphery, flagging, tag switching and long constituents (Muysken, 2000). As the structural level of CS is not of the main concern in this paper, the results will be not presented here. Based on the identified tokens of switches, two patterns of CS emerged: CS between languages and CS between writing systems. Two levels of analysis were then conducted: an analysis based on languages and the other based on writing systems. In this paper, the focus will be presenting the results of these two patterns of CS.

\subsection{RESEARCH QUESTIONS}

By examining the CS patterns in these two sets of data, this paper seeks to address the following questions:

1. What are the CS patterns between languages in email and on BBS?

2. What are the CS patterns between writing systems in email and on BBS? 
3. Do CS patterns at the two levels interact with each other in email and on BBS?

\section{FINDINGS}

Two levels of CS were identified: CS between languages (Mandarin, Taiwanese, and English) and CS between writing systems (SWC, Zhuyin, and English alphabet). Contrary to previous findings which examined only CS at the conversational level, this study found that CS can occur at these two levels. This section describes the CS patterns found in the corpus, together with the complexity of switching languages and writing systems in emails and on BBSs.

\subsection{CS BETWEEN LANGUAGES IN EMAILS}

The languages used in the emails include Mandarin, Taiwanese, and English. In terms of language choice in the emails, Table 1 shows that among the 223 emails, CS occurs in 111 emails, English only occurs in 57 emails, Mandarin only occurs in 56 emails and there is no email written in Taiwanese only. It is not surprising to find that no email is written in Taiwanese only since there is not yet a standardized written system for Taiwanese. However, the overwhelming use of English is interesting since it is not a language used in everyday conversation. Not only does CS occur in almost $50 \%$ of the emails, there are more than $25 \%$ of emails written in English only. This suggests that English is not viewed as a 'marked' language (Myers-Scotton, 1993) in online environment as opposed to face-to-face interaction. Similar to Paolillo’s (Paolillo 1999; Paolillo 2001) finding that English is predominantly used by his Indian participants interacting on Internet Relay Chat, it is found in this study that English is used in two ways: English only and CS between English and Mandarin/Taiwanese. Contrary to the common practice in face-to-face interaction in Taiwan where Mandarin and Taiwanese prevail, the email medium renders the use of English acceptable and to certain extent 'unmarked' in online environment. This indicates that email communication is viewed as a bilingual environment of at least Mandarin/English as opposed to a bilingual environment of Mandarin/Taiwanese in face-to-face interaction.

In emails where CS occurs, three patterns are identified: CS between Mandarin and Taiwanese, CS between Mandarin and English, and CS between Mandarin, Taiwanese, and English. Table 2 shows that the majority of CS occurs between Mandarin and English, with only one instance of CS between Mandarin and Taiwanese and one instance of CS between Mandarin, Taiwanese and English. As mentioned earlier, Taiwanese is not 
usually used in writing, thus accounting for the low frequency of CS between Mandarin and Taiwanese and of CS between Mandarin, Taiwanese, and English.

\begin{tabular}{|lcc|}
\hline & Number of emails & Percentage \\
\hline CS & 111 & 49.78 \\
English only & 57 & 25.56 \\
Mandarin only & 55 & 24.66 \\
Taiwanese only & 0 & 0.00 \\
Total & $\mathbf{2 2 3}$ & $\mathbf{1 0 0 . 0 0}$ \\
\hline
\end{tabular}

Table 1 Number of emails in relation to language choice

\begin{tabular}{|lcc|}
\hline & Number of emails & Percentage \\
\hline CS between M/E & 109 & 98.20 \\
CS between M/T & 1 & 0.90 \\
CS between M/T/E & 1 & 0.90 \\
Total & $\mathbf{1 1 1}$ & $\mathbf{1 0 0 . 0 0}$ \\
\hline
\end{tabular}

Table 2 Number of emails in terms of CS patterns between languages $\mathrm{M}=$ Mandarin; $\mathrm{T}=$ Taiwanese; $\mathrm{E}=$ English

The following illustrates examples of CS between Mandarin and English. Example (1) shows that CS occurs at the clausal level, where an English clause is embedded in a Mandarin-based sentence and example (2) shows an English lexical item inserted in a Mandarin-based sentence. In both examples, we can see that the main language is Mandarin, with English being inserted in the sentences. Example (3) illustrates the only case of CS between Mandarin and Taiwanese, where the Taiwanese lexical item is inserted in the Mandarin-based sentence. Example (4) illustrates CS between Mandarin, Taiwanese and English, where Mandarin is the main language with Taiwanese and English items being inserted in the sentence. In all these examples, Mandarin serves to be the main language with Taiwanese and English providing lexical items or phrases.

1) CS between Mandarin and English

\section{聽說目前最好看的是紅磨坊及珍珠港 but I like exciting one 生死極速}

'I heard that the best [movies] are Moulin Rouge and Pearl Harbor, but I like the exciting one, Fast and furious. 
2) CS between Mandarin and English

你確定是 $7 / 28$ 的 flight

'Are you sure the flight is on $28^{\text {th }}$ of July?

3) CS between Mandarin and Taiwanese

我要吃去XY冰

'I want to eat crushed ice.'

4) CS between Mandarin, Taiwanese, and English

說一些啦哩拉椬的... 心情 down 到谷底

'[he/she] said some tedious things, [which makes me feel] so down.

\subsection{CS BETWEEN WRITING SYSTEMS IN EMAILS}

When dealing with spoken conversation, previous research discusses only CS between languages or varieties of languages. However, in terms of written media, we find that CS can occur between different writing systems and that the patterns are different from those between languages. The data reveals that there are four patterns of CS between writing systems: CS between SWC and Zhuyin; CS between SWC and English alphabet; CS between English alphabet and Zhuyin; and CS between SWC, Zhuyin and English alphabet (see Table 3). Table 3 shows that CS between SWC and English alphabet occurs most frequently (68.71\%), followed by CS between SWC, Zhuyin and English alphabet (19.05\%), CS between SWC and Zhuyin (11.56\%), and finally CS between English and Zhuyin $(0.68 \%)$. In addition, Table 3 shows that Zhuyin is used quite extensively, occurring in 46 emails, taking up almost one third of the email corpus. Contrary to its main function in facilitating the acquisition of the Mandarin pronunciation, the result shows that Zhuyin can be used in writing.

\begin{tabular}{|lcc|}
\hline & Number of emails & Percentage \\
\hline CS between SWC/Z & 17 & 11.56 \\
CS between SWC/EN & 101 & 68.71 \\
CS between EN/Z & 1 & 0.68 \\
CS between SWC/Z/EN & 28 & 19.05 \\
Total & $\mathbf{1 4 7}$ & $\mathbf{1 0 0 . 0 0}$ \\
\hline
\end{tabular}

Table 3 Number of emails in terms of CS patterns between writing systems SWC = Standard Written Chinese; Z = Zhuyin; EN = English alphabet 
The following illustrates examples of CS between writing systems. Example (5) shows that CS occurs between three writing systems, SWC, Zhuyin and English alphabet. In this example, we see that the message is mainly composed of SWC, with English lexical items and phrases inserted in the sentences. A Zhuyin symbol ' $\mathrm{C}$ ', pronounced as [ग], is also inserted, functioning as a discourse marker. Example (6) is the only instance of CS between Zhuyin and English alphabet found in the email corpus. The Zhuyin symbol ' '', pronounced as [ei] functions as an attention-marker. Example (7) is a classic example of CS between SWC and Zhuyin. The main utterance is written in SWC with two Zhuyin symbols being inserted, the first one functioning as a discourse marker and the second one as an interjection. As can be seen from examples (5) to (7), Zhuyin symbols mainly function as discourse markers and interjections. The use of these symbols indicates the conversation-like nature of CMC, as found in previous studies (see e.g. Crystal, 2001). Apart from the above-mentioned patterns, CS between SWC and English alphabet accounts for the majority of the email corpus $(68.71 \%)$. Examples (8) and (9) show the pattern of CS between SWC and English alphabet. In example (8), an interjection 'anyway' is inserted at the beginning of the sentence, indicating a topic-shift. In example (9), the interjection is inserted at the end of the sentence, indicating laughter from the part of the writer.

5) CS between SWC, Zhuyin and English alphabet

llove him so much... 真的好帥卫.

'I love him so much... so handsome!'

6) CS between English and Zhuyin

1? As I remembered, Tina should start working from June 14, right?

... '[ei]? As I remembered, Tina should start working from June 14, right?'

7) CS between SWC and Zhuyin

不胖Y, 与! 很好! 高又壯!

'(he is) not fat. Very good! (he is) tall and strong!'

8) CS between SWC and English alphabet

Anyway 我們在年前有要聚餐嗎?

... 'Anyway, are we going to meet before the New Year?' 
9) CS between SWC and English alphabet

我明天先去練保齡球囉! ha...ha.a.aa...hh.a..a...

'I'll go to play bowling first tomorrow. Ha! Ha! Ha!'

Note that the number of total instances of CS in Table 3 does not correspond to that in Table 2. This can be attributed to three reasons: first, since both SWC and Zhuyin are used to represent Mandarin, the interchange between these two writing systems does not constitute CS between languages. Second, the occurrences of CS between SWC and English alphabet amount to greater number than those of CS between Mandarin and English. This can be explained by the fact that proper nouns are not included when considering CS between languages. Third, instances of interjections and discourse markers written in English alphabet (e.g. anyway and $h a$ ) are also considered as CS between writing systems (as shown in examples (8) and (9)). These findings are similar to Su's (2003) study in which she suggests that Zhuyin is often employed in online writing to indicate an informal or jocular tone as in spoken interaction. The fact that English alphabet is used for discourse particles and interjections also marks the informality of the writing style exhibited in email communication.

\subsection{CS BETWEEN LANGUAGES ON BBS}

The BBS data also identifies two levels of CS. CS between languages and CS between writing systems. As in the email corpus, the languages used on BBS include Mandarin, Taiwanese, and English. Based on these three languages, the number of CS instances between languages is illustrated in Table 4. The table shows that out of the 520 postings collected in PTT BBS, 1218 CS instances were identified; and out of the 520 postings collected in NTC BBS, 1346 CS instances were identified. In total, 2564 instances of CS were identified. These 2564 instances were then categorised according to the patterns of CS (see Table 5).

\begin{tabular}{|lcc|}
\hline & Number of postings & Tokens of CS \\
\hline PTT BBS & 520 & 1218 \\
NTC BBS & 520 & 1346 \\
Total & $\mathbf{1 0 4 0}$ & $\mathbf{2 5 6 4}$ \\
\hline
\end{tabular}

Table 4 Tokens of CS between languages 
Three patterns of CS between languages were identified: CS between Mandarin and Taiwanese, CS between Mandarin and English, CS between Mandarin, Taiwanese, and English. Table 5 presents the three patterns of CS on both PTT BBS and NTC BBS. On both BBS websites, we see that CS occurs most frequently between Mandarin and English, followed by between Mandarin and Taiwanese, and then between Mandarin, Taiwanese, and English. Considering these two BBS websites together, CS between Mandarin English accounts for more than 50\% (53.55), followed by CS between Mandarin and Taiwanese (33.42\%), and CS between Mandarin, Taiwanese and English (13.03\%). In comparison with the CS patterns in the email corpus, there are more occurrences of CS between Mandarin and Taiwanese and between Mandarin, Taiwanese and English. This difference may be explained by two aspects: one concerning the selection of participants in the two mediums and the other concerning the time of data collection. In the email corpus, participants were recruited through a social network. This means that the email language use may be restricted to this particular group. On the other hand, participants on the BBS websites do not necessarily know each other, suggesting that language use is not restricted to a single group. Instead, such language use can be interpreted as a reflection of the broader Taiwanese BBS community in comparison with the language use found in the email corpus. A second explanation accounting for the different patterns of CS in the two mediums may be related to the time of data collection. The email corpus was collected between 2001 and 2004 while the BBS corpus was collected between 2006 and 2007. The difference in patterns of CS may be due to changes in language choice and language use in online environment. That is, Taiwanese seems to be used more frequently between 2006 and 2007. Diachronic studies are required to understand how language choice and language use have changed in CMC setting in Taiwan.

\begin{tabular}{|lccccc|}
\hline & PTT BBS & Percentage & NTC BBS & Percentage & Total percentage \\
\hline CS between M/T & 362 & 29.72 & 495 & 36.78 & 33.42 \\
CS between M/E & 653 & 53.61 & 720 & 53.49 & 53.55 \\
CS between M/T/E & 203 & 16.67 & 131 & 9.73 & 13.03 \\
Total & $\mathbf{1 2 1 8}$ & $\mathbf{1 0 0 . 0 0}$ & $\mathbf{1 3 4 6}$ & $\mathbf{1 0 0 . 0 0}$ & $\mathbf{1 0 0 . 0 0}$ \\
\hline
\end{tabular}

Table 5 Patterns of CS between languages

The following shows some examples found on the BBS websites. Example (10) illustrates a Taiwanese item ‘草仔粿, Chinese rice cakes' being inserted in a Mandarin-based sentence. Example (11) shows that CS between Mandarin and English often involves the 
insertion of English lexical items. This is the most common type of switching between Mandarin and English. Example (12) is a long example involving switching from Mandarin to Taiwanese, from Taiwanese to Mandarin and then finally from Mandarin to English. The expression '焦底', literally meaning 'burnt bottom', is a common Taiwanese expression to refer to overcooked food. The English phrase at the end functions as a comment for the whole utterance.

10) CS between Mandarin and Taiwanese

\section{幾個歐巴桑正在手工現做草仔粿}

'There are several old women making some hand-made Chinese rice cakes.'

11) CS between Mandarin and English

有人可以推一下公館便宜又 NICE 的男性服飾嗎? 或是一些 BRAND 也可以

'Can anyone recommend any cheap and nice men's wear in Gongguan or just some brands [of men's wear]?'

12) CS between Mandarin, Taiwanese and English

初戀就像細火慢燉的一碗誁，可惜上桌時仍然不小心焦底. 那是整鍋丟呢?還是跳上 層的吃? To eat or not to eat, that is question.

'First love is like a bowl of slowly cooked rice soup, which is overcooked when served. So should we throw the whole thing away or should we just eat the good part? To eat or not to eat, that is the question.'

\subsection{CS BETWEEN WRITING SYSTEMS ON BBS}

Similar to the email corpus, three writing systems are found to be at use on the BBS websites: SWC, Zhuyin and English alphabet. Based on these three writing systems, the number of CS instances between writing systems is illustrated in Table 6 . The table shows that out of the 520 postings collected in PTT BBS, 1202 CS instances were identified; and out of the 520 postings collected in NTC BBS, 1305 CS instances were identified. In total, 2507 instances of CS were identified. These instances were then categorised according to the patterns of CS (see Table 7). 


\begin{tabular}{|lcc|}
\hline & Number of postings & Tokens of CS \\
\hline PTT BBS & 520 & 1202 \\
NTC BBS & 520 & 1305 \\
Total & $\mathbf{1 0 4 0}$ & $\mathbf{2 5 0 7}$ \\
\hline
\end{tabular}

Table 6 Tokens of CS between writing systems

Three patterns of CS between writing systems were identified: CS between SWC and Zhuyin, CS between SWC and English alphabet, CS between SWC, Zhuyin and English alphabet. Table 7 shows the three patterns of CS between writing systems. Table 7 shows that CS occurs most frequently between SWC and English alphabet (47.51\%), followed by between SWC and Zhuyin (38.31\%), and then between SWC, Zhuyin, and English alphabet $(14.18 \%)$ in both BBS websites. Different from the email corpus, there is no instance of CS between Zhuyin and English alphabet.

\begin{tabular}{|lcc|}
\hline & Number of postings & Tokens of CS \\
\hline PTT BBS & 520 & 1202 \\
NTC BBS & 520 & 1305 \\
Total & $\mathbf{1 0 4 0}$ & $\mathbf{2 5 0 7}$ \\
\hline
\end{tabular}

Table 7 Patterns of CS between writing systems

Examples (13) to (15) illustrate the three patterns of CS. Example (13) shows that the Zhuyin symbol 'ट', pronounced as [ग], is used as a discourse marker (see also example (5)). Considering examples (5), (7) and (13), we see that Zhuyin symbols are extensively used as discourse markers, demonstrating the spoken features of the mediums. Although the use of Zhuyin is common, the most frequently used pattern is CS between SWC and English alphabet. Example (14) illustrates a case of switching between these two writing systems. The least common type of CS is that between the three writing systems. Example (15) offers an interesting example where three writing systems are used in a sentence. In this example, the two pronouns are in English alphabet with the main verb in SWC and the discourse particle at the end of the sentence in Zhuyin. This demonstrates an example of language play by employing three writing systems.

13) CS between SWC and Zhuyin

太慢, 夠了乙

'[lt's] too slow. That's enough!' 
14) CS between SWC and English alphabet

早就 stand by 好了

'[l'm] already standing by.'

15) CS between SWC, Zhuyin and English alphabet
I 服了 you 为.
'I really admire you.'

As can be seen from Tables 1 to 7, there is no one-to-one correspondence between the two levels of CS. CS between languages does not necessarily lead to CS between writing systems and vice versa. This finding is interesting since it is often believed that CS between languages elicits CS between writing systems. However, the data reveals otherwise. For instance, when CS occurs between Mandarin and Taiwanese, this often does not lead to CS between writing systems since both languages are written in SWC (see examples (10)). When there is CS between writing systems such as SWC and Zhuyin, this does not lead to switching between languages since both systems are to represent Mandarin (see examples (7) and (13)). Moreover, CS does not occur between Taiwanese and English in both the email and BBS corpus (see Tables 2 and 5). This may be attributed to the fact that since there is no standardized writing system for Taiwanese, it is more difficult for the online users to represent the language systematically, thus opting for Mandarin, which has long been identified with SWC. Similarly, CS does not occur between Zhuyin and English alphabet (see Tables 3 and 7). This may be explained by the fact that Zhuyin is traditionally used for aiding the learning of Chinese characters and it main appears in textbooks or children's books. Further, the Zhuyin symbols are mainly used as discourse markers in conjunction with SWC as illustrated in the examples. Hence there are instances of CS between SWC, Zhuyin, and English, but not between Zhuyin and English. Finally when comparing the patterns of CS between the two mediums, we see that the patterns of CS are similar, with variations in the number of tokens in each pattern. As discussed, such differences can be explained by two factors: one concerning participants and the other concerning the time of data collection. Further research is required for comparison.

To sum up, it is found that CS is a common practice in both emails and BBS postings. Moreover, CS occurs at two levels in both CMC mediums. What is of particular interest is the fact that these two levels do not necessarily correspond to each other. That is, CS between writing systems does not guarantee CS between languages as illustrated in the 
above examples. In addition, both emails and BBS postings entail an informal writing style which fosters the use of writing systems such as Zhuyin and the combination of various writing systems. Although the CMC data are delivered in a written format, both email writers and BBS users employ a new variety known as 'talking in writing', including 'writing letters which are mailed over the telephone, (a sort of) ... panel discussion in slow motion. They use language as if they were having a 'verbal' conversation' (Spitzer, 1986, p. 19).

\section{CONCLUSION}

This paper examined two types of CMC mediums including a corpus of 223 emails and a corpus of 1040 BBS postings, focusing on CS among the Chinese-English bilinguals in Taiwan. Specifically, this paper discussed CS phenomenon at two levels: CS between languages and CS between writing systems. Three patterns of CS between languages are identified in both emails and BBS postings; and four patterns of CS between writing systems are identified in emails and three in BBS postings. It is found that CS at these two levels does not necessarily correspond to each other. That is, where there is no CS between languages, there may be CS between writing systems and vice versa. Contrary to previous research which investigated CS in spoken interaction, this study examined CS in written data, specifically on email and BBS. It is found that CMC, considered as an informal medium, often fosters creative use of writing styles and systems. The data reveals that bilinguals make use of their linguistic resources, including writing systems, in online environment in a creative way. Further research in the area of bilingual language use in online environment is required for the understanding of such widespread practices in the world.

\section{ENDNOTES}

1 The term 'bilingual' will be used to refer to both bilingual and multilingual in this paper.

2 There are different ways of referring to the alphabetical writing system such as Roman or Latin alphabet. To avoid the confusion, here in this paper, the 26 letters used to represent English are referred to as English alphabet. The term, Taiwanese, can be used to refer to both the people and the language (also known as Southern Min). In this paper, it is most often used to refer to the language.

In the following examples, Mandarin is in normal font, English is italicized and Taiwanese is underlined. In terms of writing systems, SWC is in normal font, English alphabet is italicized and Zhuyin symbols are boxed. 


\section{REFERENCES}

Auer, P. (1984). Bilingual conversation. Amsterdam: John Benjamins.

Auer, P. (ed.) (1998). Code-switching in conversation: Language, interaction and identity. London: Routledge.

Baron, N. (2000). Alphabet to email: How written English evolved and where it's heading. London: Routledge.

Chao, S. C. (2007). A study of elementary school English teachers' opinions on the first-grade English curriculum and instruction. Unpublished MA thesis, Chiayi University, Taiwan.

Chiung, W. T. (2001). Romanization and language planning in Taiwan. The Linguistic Association of Korea Journal, 9 (1), 15-43.

Clyne, M. (1987). Constraints on codeswitching: How universal are they? Linguistics, 25, 739-764.

Clyne, M. (1991). Community languages: The Australian experience. Cambridge: Cambridge University Press.

Crystal, D. (1997). English as a global language. Cambridge: Cambridge University Press.

Crystal, D. (2001). Language and the Internet. Cambridge: Cambridge University Press.

Danet, B. (2001). Cyberpl@y: Communicating online. Oxford: Berg. Available at: http://pluto.mscc.huji.ac.il/ msdanet/cyberpl@y/.

Danet, B.; Herring, S. (2003). Introduction: The multilingual internet. Journal of Computer-Mediated Communication, 9 (1). Available at: http://jcmc.indiana.edu/vol9/issue1/intro.html.

Danet, B.; Herring, S. (Eds.) (2007). The multilingual Internet: Language, culture, and communication online. New York: Oxford University Press.

Government Information Office. (2002). Information Yearbook 2000. Available at: http://www.gio.gov.tw/info/book/index.htm.

Grimes, B. (Ed.). (1996). Ethnologue (13th ed.) Dallas, TX: Summer Institute of Linguistics.

Gumperz, J. J. (1982). Discourse strategies. Cambridge: Cambridge University Press.

Haugen, E. (1953). The Norwegian language in America: A study of bilingual behavior. Philadelphia, PA: Pennsylvania University Press.

Heller, M. (Ed.). (1988). Codeswitching: Anthropological and sociolinguistic perspectives. Berlin: Mouton de Gruyter.

Henry, S. B. (2002). What is a BBS? Available at: http://www.ourhutch.com/ambassador/whatisit.htm.

Herring, S. C. (Ed.). (1996). Computer-mediated communication: Linguistic, social and cross-cultural perspectives. Amsterdam \& Philadelphia, PA: John Benjamins.

Herring, S. C. (2001). Computer-mediated Discourse. In D. Tannen, D. Schiffrin, \& H. Hamilton (Eds.), Handbook of Discourse analysis, (pp. 612-634). Oxford: Blackwell.

Herring, S. C. (2004). Computer-mediated discourse analysis: An approach to researching online behavior. In S.A. Barab, R. Kling, \& J. Gray (Eds.), Designing for virtual communities in the service of learning (pp. 338-376). Cambridge \& New York: Cambridge University Press.

Hine, C. (2000). Virtual ethnography. London: Sage. 
Hinrichs, L. (2006). Codeswitching on the web: English and Jamaican Creole in e-mail communication. Philadelphia: John Benjamins.

Hsiau, A. (1997). Language ideology in Taiwan. Journal of Multilingual and Multicultural Development, 18, 302-315.

Huang, D. L. J. (2004). Code-switching and language use in emails: A case study of a network of Chinese-English bilinguals in Taiwan. Unpublished PhD dissertation: University of Melbourne.

Huang, D. L. J. (2008). Language use and code-switching on the Internet, focusing on two selected BBSs in Taiwan. A research report sponsored by Providence University, Taiwan.

Huang, S. F. (1993). Language, society, and ethnic identity. Taipei: Crane.

Ishii, K.; Wu, C. (2006). A comparative study of media cultures among Taiwanese and Japanese youth. Telematics and Informatics, 23, 95-116.

Lam, W. S. E. (2004). Second language socialization in a bilingual chat room: Global and local considerations. Language Learning \& Technology, 8 (3), 44-65.

Milroy, L. (1987). Language and social network (2nd ed.). Oxford: Blackwell.

Milroy, L.; Muysken, P. (Eds.). (1995). One speaker, two languages: Cross-disciplinary perspectives on code-switching. New York, NY: Cambridge University Press.

Murray, D. E. (1995). Knowledge machines: Language and information in a technological society. London \& New York: Longman.

Muysken, P. (2000). Bilingual speech. Cambridge: Cambridge University

Myers-Scotton, C. (1993). Social motivations for codeswitching: Evidence from Africa. Oxford: Clarendon Press.

Myers-Scotton, C. (1998). A theoretical introduction to the markedness model. In C. Myers-Scotton (Ed.), Codes and consequences: Choosing linguistic varieties (pp.18-38). Oxford: Oxford University Press.

Paolillo, J. C. (1999). The virtual speech community: Social network and language variation on IRC. Journal of Computer-Mediated Communication, 4 (4). Available at: http://jcmc.indiana.edu./vol4/issue4/paolillo.html.

Paolillo, J. C. (2001). Language variation on Internet Relay Chat: A social network approach. Journal of Sociolinguistics, 4 (2), 180-213.

Spitzer, M. (1986). Writing style in computer conferences. IEEE Transactions of Professional Communication PC, 29 (1), 19-22.

Sproull, L. \& Kiesler, S. (1991). Connections: New ways of working in the networked organization. Cambridge, MA: MIT Press.

$\mathrm{Su}, \mathrm{H}$. Y. (2003). The Multilingual and Multi-Orthographic Taiwan-Based Internet: Creative Uses of Writing Systems on College-Affiliated BBSs. Journal of Computer-Mediated Communication, 9 (1). Available at: http://jcmc.indiana.edu/vol9/issue1/su.html.

Tiu, H. K. (1998). Writing in two scripts: A case study of digraphia in Taiwanese. Written Language and Literacy, 1 (2), 225-247. 
Warschauer, M. (2006). Networking the Nile: Technology and professional development in Egypt. In J. Inman \& B. Hewett (Eds.), Technology and English studies: Innovative professional paths (pp.163-172). Mahwah, NJ: Lawrence Erlbaum.

Warschauer, M.; El Said, G. R.; Zohry, A. (2002). Language choice online: Globalization and identity in Egypt. Journal of Computer-mediated Communication, 7 (4). Available at: www.ascusc.org/jcmc/vol7/issue4.

Wikipedia. (2007). Chinese input methods for computers. Retrieved January 03, 2004 from http://en.wikipedia.org/wiki/Chinese_input_methods_for_computers.

Cite this article as: Huang, Daphne Li-jung. (2009). Language use in asynchronous computermediated communication in Taiwan. Australian Review of Applied Linguistics 32 (2), 12.1-12.22. DOI: $10.2104 /$ aral0912. 